\title{
Hydromechanics and Kinematics in Preferential Flow
}

\author{
Peter F. Germann
}

\begin{abstract}
:
Preferential flow covers macropore flow, nonequilibrium flow, and finger flow that are here exclusively approached with gravity-driven viscous flow. The basic unit is a water content wave whose two parameters are the wave's film thickness and its mainly vertical contact area per unit soil volume. The spatiotemporal wave properties depend on soil structure and on the intensity and duration of water input to the surface. Kinematic wave theory provides the mathematical tool for solving the analytical expressions. Three cases are the basic building blocks for approaching preferential flow: (a) single pulse, (b) a faster pulse trails a slower pulse, and $(c)$ a faster pulse overtakes a slower pulse. Analytical procedures are presented for each case, and the potential of their applications is discussed. The analytical expressions and the superfluous representative elementary volume greatly facilitate preferential flow modeling.
\end{abstract}

Key Words: Darcy's law, permeable media, preferential flow, viscous flow

(Soil Sci 2018;183: 1-10)

referential flow $(\mathrm{PF})$ in soils and similar permeable media is fast and gravity driven, and only a minor fraction of porosity participates in it. According to Jarvis et al. (2016), PF embraces unstable finger flow, macropore flow, and nonequilibrium flow. Hence, PF opposes ordinary flow that supposedly is stable and homogeneous and is in equilibrium with capillarity, that is, Richards' (1931) capillary flow (CF). Although opposing $\mathrm{CF}$, most approaches to $\mathrm{PF}$ still circle around $\mathrm{CF}$ and have not yet evolved as independent flow types at levels comparable with CF. As presented here, however, viscous flow (VF) in permeable media is independent from $\mathrm{CF}$, while capillarity appears merely as abstractor of water from VF. One-dimensional Darcy (1856) and two-dimensional Dupuit (1863)-Forchheimer approaches to flow in saturated permeable media as well as Hagen-Poiseuille (1846) flow in thin tubes are based on Newton's law of shear. The same principle is here applied to transient flow in partially water-saturated permeable media.

The article introduces first the concept of $\mathrm{PF}$ and its delineation from ordinary, that is, Richards' (1931) CF. It then presents the basic analytical expressions for VF in permeable media, whereas the more vivid kinematic-wave theory provides for the general mathematical solutions.

\section{FACETS OF PREFERENTIAL FLOW AND ITS DISTINCTION FROM CAPILLARY FLOW}

In the mid-19th century, physicians and physiologists got interested in the physics of blood flow: Hagen and Poiseuille (i.e., Poiseuille, 1846) applied Newton's (1729) law of shear to laminar flow in thin tubes. Moreover, numerous engineering projects triggered hydraulic approaches to flow in saturated permeable media: Darcy (1856), designing filters for the public water supply of Dijon, investigated onedimensional flow in water-saturated permeable media, whereas Dupuit (1863) presented two-dimensional groundwater flow toward ditches and wells. Darcy's (1856) law and therefore Dupuit's (1863) approach are also based on Newton's law of shear as will be presented later on. Observations on water and solute transport in partially saturated soils were mainly based on lysimeter studies, like those of Lawes

University of Bern, Bern, Switzerland.

Address for correspondence: Dr. Peter F. Germann, Geographisches Institut der Universität Bern, Hallerstrasse 12, CH-3012 Bern, Switzerland.

E-mail:pf.germann@bluewin.ch

Financial Disclosures/Conflicts of Interest: None reported.

Received February 15, 2018.

Accepted for publication June 7, 2018.

Copyright (O) 2018 Wolters Kluwer Health, Inc. All rights reserved.

ISSN: 0038-075X

DOI: $10.1097 / S S .0000000000000226$ et al. (1882), for instance, who anticipated PF. They reported from the Rothamsted (UK) research station that "The drainage water of a soil may thus be of two kinds (1) of rainwater that has passed with but little change in composition down the open channels of the soil or (2) of the water discharged from the pores of a saturated soil." Further, "The respective proportions of direct and general drainage will vary much in different soils and under different circumstances." Also, "The two kinds of drainage water here mentioned differ much in composition, the direct channel drainage containing a much smaller proportion of soluble salts than is found in the true discharge from the soil."

Steps leading to this presentation include the lateral Br-sorption from macropores into tinier pores (Germann et al., 1984) that hints at the priority of PF over CF. Further, Germann (1986) concluded from infiltration-drainage measurements in the Coshocton lysimeters (Harrold and Dreibelbiss, 1958; Kelley et al. 1975) that precipitations of $10 \mathrm{~mm} / \mathrm{d}$ were sufficient for drainage to respond at the 2.5-m depth within 1 day. This results in wetting front velocities of approximately $2 \times 10^{-5} \mathrm{~m} \cdot \mathrm{s}^{-1}$. In the Kiel sand tank, Germann and al-Hagrey (2008) noted that the capillary potential $\psi$ Pa collapsed close to atmospheric pressure during fast infiltration with a wetting front velocity of $3.3 \times 10^{-5} \mathrm{~m} \cdot \mathrm{s}^{-1}$. Nimmo (2012) suggested that PF also occurs under nonsaturated conditions, whereas Germann (2018a) provided experimental evidence of shock-like infiltration to occur in partially water-saturated permeable media under nearatmospheric pressure if the wetting shock front remains connected with the surface.

Preferential flow is usually associated with macropores, which implicitly call for the remaining pore space as micropores. The resulting pore space dichotomy requires demarcation. Jarvis et al. (2016), for instance, consider pores wider than approximately 300 to $500 \mu \mathrm{m}$ as macropores. However, despite the majority of such qualitative demarcations, their quantification becomes essential when, out of opposition to CF, separate approaches to flow are to be applied to each pore class. In order to avoid all together the arbitrarily set thresholds and associated flow processes, the term permeable media is here given preference over porous media. Permeable media are thought of solids that are penetrated by voids such as fissures, cracks, and pores that are able to conduct water without restrictions on either the geometry or the volumetric share of the voids. Likewise, the proposed flow process does not require any a priori restriction with the exception of depending on low Reynolds numbers.

Despite the general recognition that PF is fast, there are few studies expressively dealing with its velocity. Ignoring capillarity, Beven and Germann (1981) approached gravity-driven and laminar macropore flow with VF according to Hagen-Poiseuille (1846). Beven and Germann (1981) also considered the theory of kinematic waves (KW) according to Lighthill and Witham (1955) as a suitable mathematical tool for dealing with VF along presumed macropores. 
The modeling study also revealed that the widest water-conducting paths dominate flow so strongly that already slightly narrower conduits markedly lose influence on flow. Germann (1985) demonstrated experimentally the feasibility of KW theory to the parameterization of $\mathrm{PF}$ in a block of polyester-cemented sand. Further investigations, for instance, those of Germann and al-Hagrey (2008), Hincapié and Germann (2009a, b; 2010), Germann and Karlen (2016), and Germann and Prasuhn (2017), led to the in situ experimental parameterization of VF. Nimmo's (2010) sourceresponsive free-surface film flow considers various elements within the same theoretical framework.

\section{BASICS OF VISCOUS FLOW IN PERMEABLE MEDIA}

\section{Newton's Law of Shear}

A rectangular pulse $P\left(q_{S}, T_{B}, T_{E}\right)$ provides the input to the soil surface, where $q_{S} \mathrm{~m} \cdot \mathrm{s}^{-1}$ is its volume flux density (the subscript $S$ refers to the surface), whereas $T_{B}$ and $T_{E}$ (both $\mathrm{s}$ ) are the times of the pulse's beginning and ending. At $T_{B}, P$ presumably initiates a water film gliding down the permeable medium. According to Fig. 1, the thickness $F$ and the specific horizontal contact length $L \mathrm{~m} \cdot \mathrm{m}^{-2}$ per cross-sectional area $A \mathrm{~m}^{2}$ of the permeable medium define the film, whereas $f$ is the thickness variable, and $\mathrm{d} f$ is the thickness of a layer (lamina in Latin, hence laminar flow). The solid-water interface (SWI) at $f=0$ and the air-water interface (AWI) at $f=F$ confine the film. The film is accelerated by the specific weight $\rho \times g \mathrm{~N} \cdot \mathrm{m}^{-3}$, where $\rho\left(=1,000 \mathrm{~kg} \cdot \mathrm{m}^{-3}\right)$ is the water's density, and $g\left(=9.81 \mathrm{~m} \cdot \mathrm{s}^{-2}\right)$

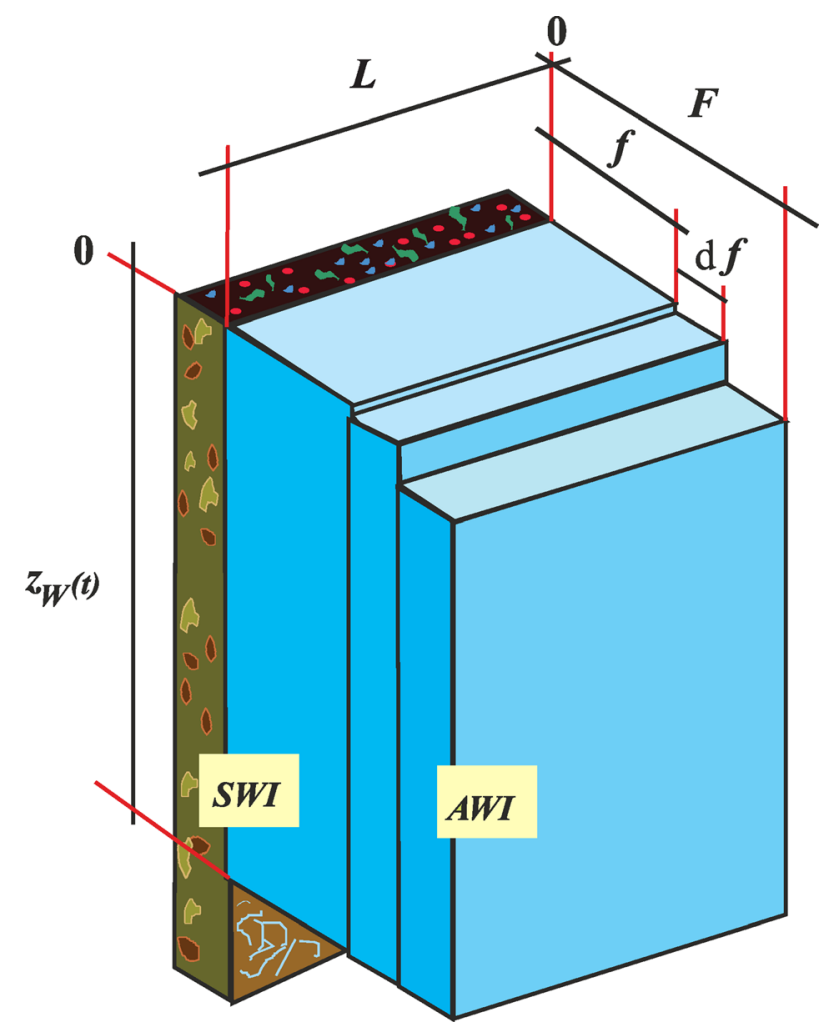

FIGURE 1. Film flow along a vertical plane. $F$ is film thickness, $f$ the thickness variable, and $d f$ the lamina thickness; $z_{w}(t)$ is the vertical position of the wetting shock front as function of time $t ; L$ is the specific contact line of the film per unit cross-sectional area; AWI and SWI are the interfaces between water and air as well as water and solid, respectively. Adapted with permission from Germann (2014). A color version of this figure is available in the online version of this article. [unl color is acceleration due to gravity. The shear force $\varphi \mathrm{Pa}$ acts in the direction opposite to gravity, thus decelerating the film such that it moves downward with a constant velocity $v_{W} \mathrm{~m} \cdot \mathrm{s}{ }^{-1}$ of the wetting shock front, whereas $z_{W}(t)$ is the wetting shock front's position as function of time. The term wetting shock front indicates the flow discontinuity at $z_{W}(t)$ that is initiated at $T_{B}$ when $P$ hits the soil surface. The $z$-coordinate points is positive from the surface down. The specific contact area of the film per volume of soil amounts to $L \times A \times z_{W}$ $(t) /\left[A \times z_{W}(t)\right]=L \mathrm{~m}^{2} \cdot \mathrm{m}^{-3}$.

Momentum flux density at $f$ is $\rho \times g \times z_{W}(t) \mathrm{Pa}$ and is active in the direction of flow. Newton (1729) proposed the shear force $\varphi$ as "The resistance, arising from the want of lubricity in the parts of a fluid, is, caeteris paribus, proportional to the velocity with which the parts of the fluid are separated from each other." Thus, momentum dissipation toward the SWI is proportionate to the velocity gradient at $f$, that is, $\mathrm{d} c / \mathrm{d} f \mathrm{~s}^{-1}$, where the celerity $c \mathrm{~m} \cdot \mathrm{s}^{-1}$ is the velocity of a particular film property. The factor of proportionality is the temperature-dependent kinematic viscosity $\eta\left(\approx 10 \mathrm{~m}^{2} \cdot \mathrm{s}^{-1}\right)$. Momentum dissipation toward the SWI produces the shear force $\varphi(f) \mathrm{Pa}$ that acts in the opposite direction of momentum flux density due to gravity; $\varphi(f)$ balances the weight of the water film between $f$ and $F$ according to

$$
\varphi(f) \cdot L=\left.\eta \cdot \rho \cdot \frac{\mathrm{d} c}{\mathrm{~d} f}\right|_{f} \cdot L=\rho \cdot g \cdot L \cdot(F-f)
$$

The center part of Eq. [1] represents the dissipation of momentum due to the celerity gradient of $\mathrm{d} c / \mathrm{d} f$ at $f$. The right hand side of Eq. [1] represents the weight of the water film with the volume of $L \times(F-f)$. The dynamic force balance in Eq. [1] produces the constant celerity $c$ at $f$. Simplifying Eq. [1], separating the variables and integrating it from $f=0$ to $f=F$ under the consideration of $c(0)=0$ (the nonslip condition) yield the parabolic celerity profile in the horizontal $f$ direction as

$$
c(f)=\frac{g}{\eta} \cdot\left(F \cdot f-\frac{f^{2}}{2}\right)
$$

\section{Viscous Flow in Permeable Media}

The pulse $P$ hitting the surface at $t=T_{B}$ releases a water content wave (WCW) at $z=0$. Figure 2 depicts the WCW that envelops the spatiotemporal distribution of the mobile water content, $w(z, t)$ $\mathrm{m}^{3} \cdot \mathrm{m}^{-3}$. During input, $T_{B} \leq t \leq T_{E}$, the WCW assumes the shape of a water film according to Fig. 1. Both parameters, $F$ and $L$, are due to $P$ and the actual specific properties of the permeable medium. Cessation of $P$ at $t=T_{E}$ initiates the film's thinning.

During infiltration, that is, $T_{B} \leq t \leq T_{E}$, the specific volume $V_{\mathrm{WCW}} \mathrm{m}$ of the film as function of time is

$$
V_{W C W}(t)=F \cdot L \cdot z_{W}(t)=q_{S} \cdot\left(t-T_{B}\right)
$$

Under the auspice of $P$ and after infiltration has ceased, that is, $t>T_{E}$, the total and maximum specific water volume of the WCW remains at $V_{\text {tot }}=q_{S} \times\left(T_{E}-T_{B}\right)$ if the WCW neither gains nor loses water. The mobile water content $w \mathrm{~m}^{3} \cdot \mathrm{m}^{-3}$ amounts to

$$
w=F \cdot L
$$

The differential volume flux density at $f$ is

$$
\left.\mathrm{d} q\right|_{f}=L \cdot \mathrm{d} f \cdot c(f)
$$

Its integration from the SWI to the AWI leads to the volume flux density $q_{S} \mathrm{~m} \cdot \mathrm{s}^{-1}$ of the film as

$$
q_{S}=F^{3} \cdot L \cdot \frac{g}{3 \cdot \eta}=w_{S}{ }^{3} \cdot \frac{g}{3 \cdot L^{2} \cdot \eta}
$$




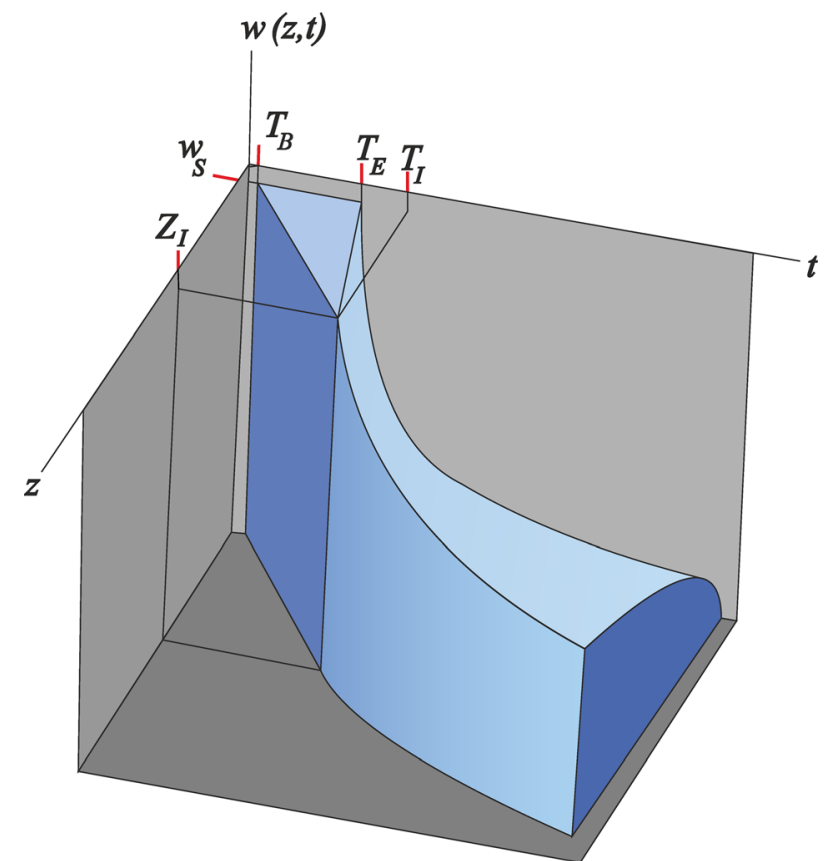

FIGURE 2. Schematic representation of a WCW. $w(z, t)$ is mobile water content, tand $z$ are the axes of time and depth; $T_{\mathrm{B}}$ and $T_{\mathrm{E}}$ indicate beginning and ending of the water pulse $P\left(q_{\mathrm{S}}, T_{\mathrm{B}}, T_{\mathrm{E}}\right)$ that hits the surface at $z=0 ; T_{1}$ and $Z_{1}$ are time and depth of the wetting front intercepting the draining front. The line from $(0,0)$ to $\left(Z_{1}, T_{1}\right)$, and beyond represents the position of the wetting shock front, $Z_{W}(t)$, whereas the line from $\left(0, T_{E}\right)$ to $\left(Z_{1}, T_{1}\right)$ gives the position of the draining front, $z_{D}(t)$. Adapted with permission from Germann (2014). A color version of this figure is available in the online version of this article. fullcolor

From the volume balance follows the velocity $v_{W} \mathrm{~m} \cdot \mathrm{s}{ }^{-1}$ of the wetting shock front as

$$
v_{W}=\frac{q_{\mathrm{S}}}{w_{\mathrm{S}}}=\frac{z_{W}(t)}{t-T_{B}}=F^{2} \cdot \frac{g}{3 \cdot \eta}
$$

Thus, the force balance, Eq. [1], leads to a constant velocity $v_{W}=\mathrm{d} z_{W}(t) / \mathrm{d} t \mathrm{~m} \cdot \mathrm{s}^{-1}$ of the film's wetting shock front. Laminar VF applies to Reynolds numbers $R e<\approx 3$, with $R e$ defined as

$$
R e=\frac{F \cdot v}{\eta}=\frac{F^{3} \cdot g}{3 \cdot \eta^{2}}=\left(\frac{3 \cdot v^{3}}{g \cdot \eta}\right)^{1 / 2} \leq 3
$$

that limits the maximal tolerable film thickness $F$ to approximately $100 \mu \mathrm{m}$

Viscous flow occurs in unsaturated permeable media, that is, $w<(\varepsilon-\theta)$, where $\varepsilon$ and $\theta$ (both $\left.\mathrm{m}^{3} \cdot \mathrm{m}^{-3}\right)$ are porosity and antecedent volumetric water content of the medium, respectively. According to Eq. [1], the pressure gradient from VF is $\Delta p /(\Delta z \times \rho \times g)=1$. Darcy's law for vertical flow is based on the same principles but for saturated media with $w=(\varepsilon-\theta)$ and the pressure gradient of $\Delta p /(\Delta z \times \rho \times g)>1$. The particular case is the hydraulic conductivity $K \mathrm{~m} \cdot \mathrm{s}^{-1}$ amounting to $q_{S}$ according to Eq. [6] under the special conditions of $w=(\varepsilon-\theta)$ and $\Delta p /(\Delta z \times \rho \times g)=1$.

The cessation of input at $T_{E}$ causes the mobile water content at the surface to jump from $w_{S}$ to 0 and the film thickness from $F$ to 0 . Therefore, the jump releases at once the rear ends of all the laminae that continue to glide one over the other. Each rear end moves with the celerity $c$ $(f)$ that reduces with decreasing distance from the SWI according to
Eq. [2]. Thus, the film starts to flatten, and the spatiotemporal distribution of the mobile water content $w(z, t)$ of a WCW after $t>T_{E}$ becomes

$$
w(z, t)=L \cdot\left(\frac{\eta}{g}\right)^{1 / 2} \cdot z^{1 / 2} \cdot\left(t-T_{E}\right)^{-1 / 2}
$$

(Germann and Karlen, 2016). The outermost lamina at $F$ moves the fastest with the celerity $c_{\mathrm{D}} \mathrm{m} \cdot \mathrm{s}^{-1}$ of the draining front (indexD), thus

$$
c_{D}=\frac{\mathrm{d} q_{S}}{\mathrm{~d} w}=3 \cdot v_{W}
$$

Equation [10] applies to all the lamina originating at $F$ during $T_{B} \leq t \leq T_{E}$; however, the slower moving wetting shock front, Eq. [7], continuously intercepts them.

Because of $c_{\mathrm{D}}=3 v_{W}$, the wetting shock front that was released at $T_{B}$ intercepts at depth and time $Z_{\mathrm{I}} \mathrm{m}$ and $T_{\mathrm{I}} \mathrm{s}$, the faster-moving draining front that was later released at $T_{\mathrm{E}}$. Depth and time of interception follow from the two relationships of $Z_{I}=\left(T_{I}-T_{B}\right) \cdot v_{W}$ and $Z_{I}=\left(T_{I}-T_{E}\right) \cdot c_{\mathrm{D}}$ that are to be solved for $Z_{\mathrm{I}}$ and $T_{\mathrm{I}}$, yielding

$$
\begin{aligned}
& Z_{\mathrm{I}}=\frac{c_{D}}{2} \cdot\left(T_{E}-T_{B}\right) \\
& T_{I}=\frac{1}{2} \cdot\left(3 \cdot T_{E}-T_{B}\right)
\end{aligned}
$$

where $T_{\mathrm{I}}$ depends only on $T_{B}$ and $T_{E}$.

After $t \geq T_{\mathrm{I}}$ and beyond $z \geq Z_{\mathrm{I}}$, the wetting shock front forms a crest (index CR) that moves downward with the decreasing velocity of

$$
v_{C R}(t)=v_{W} \cdot\left(\frac{T_{E}-T_{B}}{2 \cdot\left(t-T_{E}\right)}\right)^{2 / 3}
$$

The mobile water content at the crest declines with time according to

$$
w_{C R}(t)=w_{S} \cdot\left(\frac{T_{E}-T_{B}}{2 \cdot\left(t-T_{E}\right)}\right)^{1 / 3}
$$

Equations [13] and [14] are according to Germann (2014) and to Germann and Karlen (2016). The following three sections present the three projections of the WCW, Fig. 2, onto the $w-z$, the $w-t$, and the $z$ - $t$ planes.

\section{PROFILES OF MOBILE WATER CONTENTS $w(z, \tau)$}

The profiles of $w(z, \tau)$ are now considered that appear as projections of the WCW onto the $w-z$ plane in Fig. 2. Three intervals are to be distinguished: (i) $\left[T_{B} \leq \tau_{1} \leq T_{E}\right]$, (ii) $\left[T_{E} \leq \tau_{2} \leq T_{\mathrm{I}}\right]$, and (iii) $\left[\tau_{3} \geq T_{\mathrm{I}}\right]$. Interception time $T_{\mathrm{I}}$ is according to Eq. [12]. Figure 3 provides examples of $w(z, \tau)$ during the three periods and at $T_{\mathrm{I}}$.

\section{Interval (i): $T_{B} \leq \tau_{1} \leq T_{E}$}

The position $z_{W}\left(\tau_{1}\right)$ of the wetting shock front is

$$
z_{W}\left(\tau_{1}\right)=v_{W} \cdot\left(\tau_{1}-T_{B}\right)
$$

whereas the mobile water content is

$$
w\left(z, \tau_{1}\right)=w_{S}=F \cdot L
$$

Steady state prevails during this interval. Line $\tau_{1} / T_{\mathrm{I}}=0.24$ in Fig. 3 illustrates $w\left(z, \tau_{1}\right)$.

\section{Interval (ii): $T_{E} \leq \tau_{2} \leq T_{1}$}

The position of the wetting shock and draining fronts, $z_{W}\left(\tau_{2}\right)$ and $z_{D}$ $\left(\tau_{2}\right)$, are 


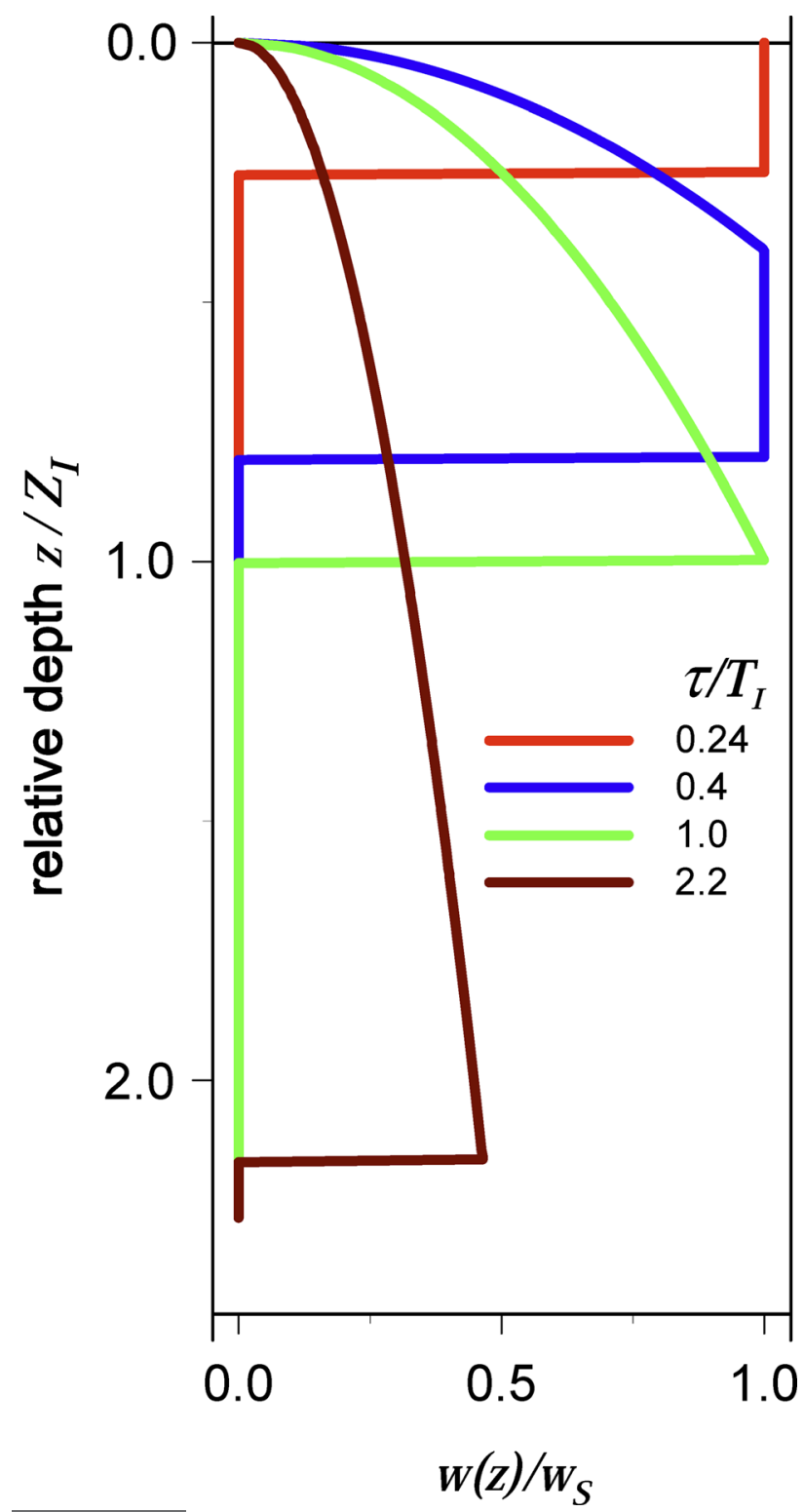

FIGURE 3. Profiles of relative mobile water contents $w(z) / w_{S}$ at the four relative times $\tau / T_{1}$ of $\tau_{1} / T_{1}=0.24, \tau_{2} / T_{1}=0.4, T_{1}=1.0$, and $\tau_{3} / T_{1}=2$.2. Adapted with permission from Germann (2014). A color version of this figure is available in the online version of this article. futc color

$$
\begin{gathered}
z_{W}\left(\tau_{2}\right)=v_{W} \cdot\left(\tau_{2}-T_{B}\right) \\
z_{D}\left(\tau_{2}\right)=c_{D} \cdot\left(\tau_{2}-T_{E}\right)
\end{gathered}
$$

The mobile water content $w\left(z, \tau_{2}\right)$ in the depth range of $0<z<z_{D}\left(\tau_{2}\right)$ amounts to

$$
w\left(z, \tau_{2}\right)=L \cdot\left(\frac{\eta}{g}\right)^{1 / 2} \cdot z^{1 / 2} \cdot\left(\tau_{2}-T_{E}\right)^{-1 / 2}
$$

In section $z_{D}\left(\tau_{2}\right)<z<z_{W}\left(\tau_{2}\right)$, it is

$$
w\left(z, \tau_{2}\right)=w_{\mathrm{S}}
$$

Line $\tau_{2} / T_{\mathrm{I}}=0.4$ in Fig. 3 illustrates $w\left(z, \tau_{2}\right)$
Interval (iii): $\tau_{3} \geq T_{\text {, }}$

The position of the wetting shock front $z_{W}\left(\tau_{3}\right)$ after interception becomes

$$
z_{W}\left(\tau_{3}\right)=c_{D} \cdot\left(\frac{T_{E}-T_{B}}{2}\right)^{2 / 3} \cdot\left(\tau_{3}-T_{\mathrm{E}}\right)^{1 / 3}
$$

(Germann and Karlen, 2016). The mobile water content in the section of $0<z<z_{W}\left(\tau_{3}\right)$ amounts to

$$
w\left(z, \tau_{3}\right)=L \cdot\left(\frac{\eta}{g}\right)^{1 / 2} \cdot z^{1 / 2} \cdot\left(\tau_{3}-T_{E}\right)^{-1 / 2}
$$

Line $\tau_{3} / T_{I}=2.2$ in Fig. 3 illustrates $w\left(z, \tau_{3}\right)$.

\section{Discussion of $w$ Profiles}

So far, the section considered only the mobile water contents $w$ $\left(z, \tau_{1,2,3}\right)$. The respective volume flux densities follow from Eq. [8]. Water balance calculations of the entire $\mathrm{WCW}$ require $w(z, t)$ profiles. Germann and Prasuhn (2017) provide examples of VF propagations in a weighing lysimeter.

\section{TIME SERIES OF MOBILE WATER CONTENTS $w(\zeta, t)$}

This section considers time series of $w(z, t)$ that show as projections of the WCW onto the $w$ - $t$ plane in Fig. 2 . Two sections are to be considered: (i) $\left[0 \leq \zeta_{1} \leq Z_{\mathrm{I}}\right]$ and (ii) $\left[\zeta_{2} \geq Z_{\mathrm{I}}\right]$, whereas the interception depth, $Z_{\mathrm{I}}$, is according to Eq. [11].

\section{Section (i): $0 \leq \zeta_{1} \leq Z_{1}$}

According to Eqs. [7] and [10], the time lapses $t_{W}\left(\zeta_{1}\right)$ and $t_{D}\left(\zeta_{1}\right)$ for the wetting shock and draining fronts to arrive at $\zeta_{1}$ are

$$
\begin{gathered}
t_{W}\left(\zeta_{1}\right)=T_{B}+\frac{\zeta_{1}}{v_{W}} \\
t_{D}\left(\zeta_{1}\right)=T_{E}+\frac{\zeta_{1}}{c_{D}}
\end{gathered}
$$

The associated mobile water contents amount to

$$
\begin{gathered}
T_{\mathrm{B}} \leq t \leq t_{W}\left(\zeta_{1}\right): w\left(\zeta_{1}, t\right)=0 \\
t_{W}\left(\zeta_{1}\right) \leq t \leq t_{D}\left(\zeta_{1}\right): w\left(\zeta_{1}, t\right)=w_{S}
\end{gathered}
$$

Equation [26] indicates steady state, that is, piston flow.

$$
t \geq t_{D}\left(\zeta_{1}\right): w\left(\xi_{1}, t\right)=w_{S} \cdot\left(\frac{t_{D}\left(\zeta_{1}\right)-T_{E}}{t-T_{E}}\right)^{1 / 2}
$$

Lines $\zeta_{1} / Z_{I}=0.4$ and 0.85 in Fig. 4A represent Eqs. [23] to [27], whereas the corresponding lines in Fig. $4 \mathrm{~B}$ show the associated volume flux densities.

\section{Section (ii): $\zeta_{2} \geq Z_{1}$}

The WCW reduces to a crested wave. According to Eq. [21], the time $t_{C R}\left(\zeta_{2}\right)$ lapsed for the wetting shock to move to $\zeta_{2}$ is

$$
t_{C R}\left(\zeta_{2}\right)=T_{E}+\left(\frac{\zeta_{2}}{c_{D}}\right)^{3} \cdot\left(\frac{T_{E}-T_{B}}{2}\right)^{-2}
$$

The mobile water content at the crested wetting shock front amounts to

$$
w_{C R}\left(\zeta_{2}, t\right)=w_{S} \cdot\left(\frac{T_{E}-T_{B}}{2 \cdot\left(t-T_{E}\right)}\right)^{1 / 3}
$$

Thus, the associated mobile water contents amount to 


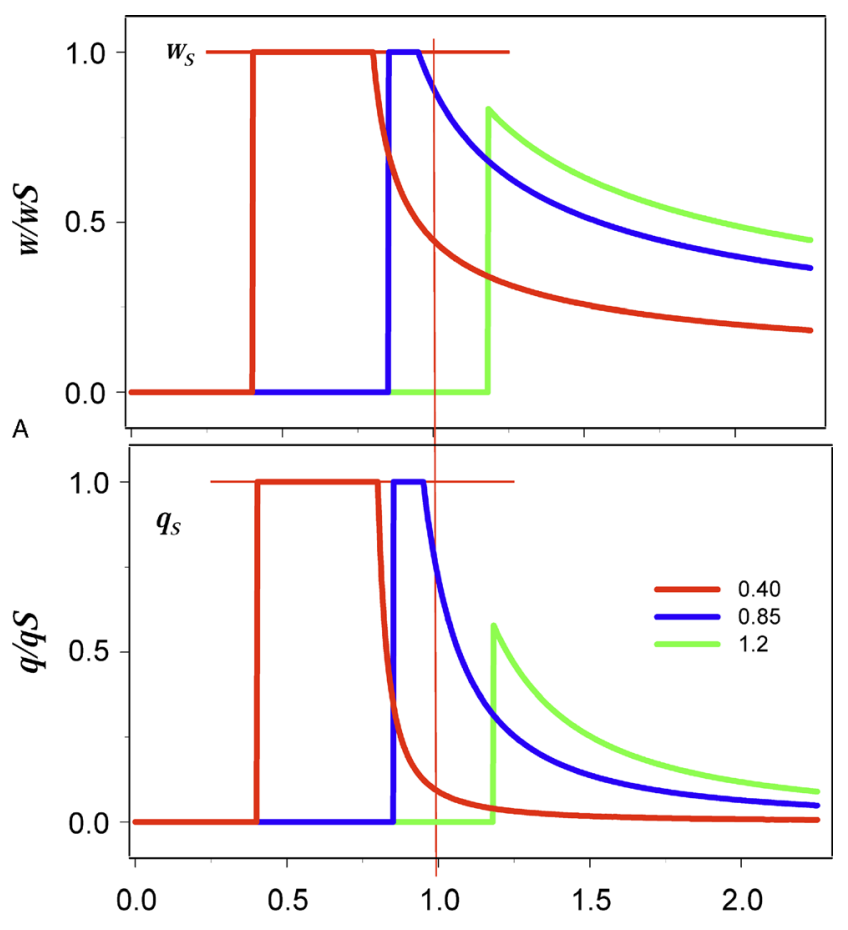

B

relative time $t / T_{I}$

FIGURE 4. Series of mobile water contents and associated volume flux densities. A, Relative mobile water content, $w / w_{\mathrm{S}}$, versus relative time $t / T_{1}$ at relative depths of $\zeta_{1} / Z_{1}=0.4$ and 0.85 , and $\zeta_{2} / Z_{1}=1.2$. B, Relative volume flux density, $q / q_{\mathrm{S}}$, versus relative time $t / T_{1}$ at the same relative depths as Fig. 4A. Adapted with permission from Germann (2014). A color version of this figure is available in the online version of this article. [ut colorer

$$
\begin{gathered}
T_{B} \leq t \leq t_{C R}\left(\zeta_{2}\right): w\left(\zeta_{2}, t\right)=0 \\
t \geq t_{C R}\left(\zeta_{2}\right): w\left(\zeta_{2}, t\right)=w_{C R}\left(\zeta_{2}, t\right) \cdot\left(\frac{t_{C R}(\zeta)-T_{E}}{t-T_{E}}\right)^{1 / 2}
\end{gathered}
$$

Line $z 2 / Z_{I}=1.2$ in Fig. 4A represents Eqs. [28] to [31]. Figure 4B depicts the volume flux densities that follow from the application of Eq. [6] to the mobile water contents.

\section{Discussion of $\boldsymbol{w}$ Time Series}

Time series of $w$ at particular depths are the favorite mode for in situ estimations of $F$ and $L$, for instance, with rapid $\theta$ measurements using time-domain reflectometry equipment (Germann, 2018b; Germann and Karlen, 2016). In order to reduce the number of variables, experimenters are advised to restrict the measurements to the depth range of $0 \leq z \leq Z_{\mathrm{I}}$. The requirement is easy to achieve by simply extending the duration of experimental input long enough, as Eqs. [11] and [12] reveal.

\section{KINEMATIC WAVE THEORY: THE TRAJECTORIES OF MOBILE WATER CONTENTS IN THE $z$ - $t$ PLANE}

So far, a single rectangular pulse $P$ was considered as input to the permeable medium that was routed according to the rules of VF. However, a more realistic scenario to natural processes requires the temporal variation of input. For that, any water input to the surface as function of time is divided into a series of $n$ rectangular pulses $P\left(T_{B}, T_{E}, q_{S}\right)_{j}$ with $1 \leq j \leq n$ that need to be routed according to VF. The selection of $\left(T_{B}, T_{E}\right)_{j}$ allows for smooth adjustment of the
VF approach to reality. A straightforward procedure of pulse routing evolves from the projection of the WCW onto the $z$ - $t$ plane leading to the temporal positions of $w(z, t)$ and, subsequently, of $q(z, t)$. The procedure draws from Lighthill and Witham (1955), who developed the KW theory for flows of water in long uniform channels and for traffic flows. Kinematic wave permits the routing of pulse series, in those each pulse carries its own duration and $w_{S}\left(q_{S}\right)$ value. The temporal positions of $w$ follow from relationships that depend exclusively on the volume balance, hence, the adjective "kinematic". The resulting analytical equations greatly facilitate modeling of PF as laminar VF. Pulse routing relies on three categories of WCW projections onto the $z-t$ plane: (i) The already known wetting- and draining-front positions, (ii) the characteristics as straight-lined trajectories of properties, such as draining fronts, which move with constant celerities, and (iii) the interception function.

Kinematic wave theory applies to VF under the premises of low Reynolds numbers, Eq. [8], and of

$$
q(w)=b \cdot w^{3}
$$

where the conductance $b \mathrm{~m} \cdot \mathrm{s}^{-1}$ amounts to

$$
b=\frac{g}{3 \cdot \eta \cdot L^{2}}
$$

Equation [32] is referred to as the VF function from which follows that

$$
w=\left(\frac{q}{b}\right)^{1 / 3}
$$

Further, VF in a particular permeable medium presumably follows the same paths independently from the input rate. This requires a constant specific surface area $L$, that is,

$$
\mathrm{d} L / \mathrm{d} q=0
$$

The constraint of Eq. [35] is referred to as macropore-flow restriction that leads to $\mathrm{d} b / \mathrm{d} q=0$ in Eqs. [32] to [34]. From the volume balance follow the wetting front velocity as

$$
v_{W}=\frac{q_{S}}{w_{S}}=b \cdot w_{S}^{2}
$$

whereas the celerity of the draining front is

$$
c_{D}=\frac{\mathrm{d} q_{S}}{\mathrm{~d} w_{S}}=3 \cdot b \cdot w_{S}^{2}
$$

Moreover, the macropore-flow restriction relates the velocity of the wetting shock front with the volume flux density of input as

$$
v_{W}\left(q_{S}\right)=b^{1 / 3} \cdot q_{S}^{2 / 3}
$$

Figure 5 illustrates the VF function, Eq. [32], and KW properties under the auspice of Eq. [35]. Thus, the slopes of lines (4) and (5) in Fig. 5 depict $v_{\mathrm{W} 1,2}$ for two mobile water contents $w_{1}$ and $w_{2}>w_{1}$ or their two corresponding volume flux densities $q_{1}$ and $q_{2}>q_{1}$. The slopes of the tangents to $q\left(w_{1}, w_{2}\right)$, lines (2) and (3) in Fig. 5 represent $c_{\mathrm{D} 1,2}$, where generally $c_{\mathrm{D}}=3 \times v_{W}$.

Three canonical cases emerge as the basic entity for approaching VF with KW theory:

(i) routing of a single pulse;

(ii)) routing of a faster pulse with higher-volume flux density that trails a slower pulse with lower-volume flux density;

(iii) routing of a faster pulse with higher-volume flux density that superimposes (overtakes) a slower pulse with lower-volume flux density. 


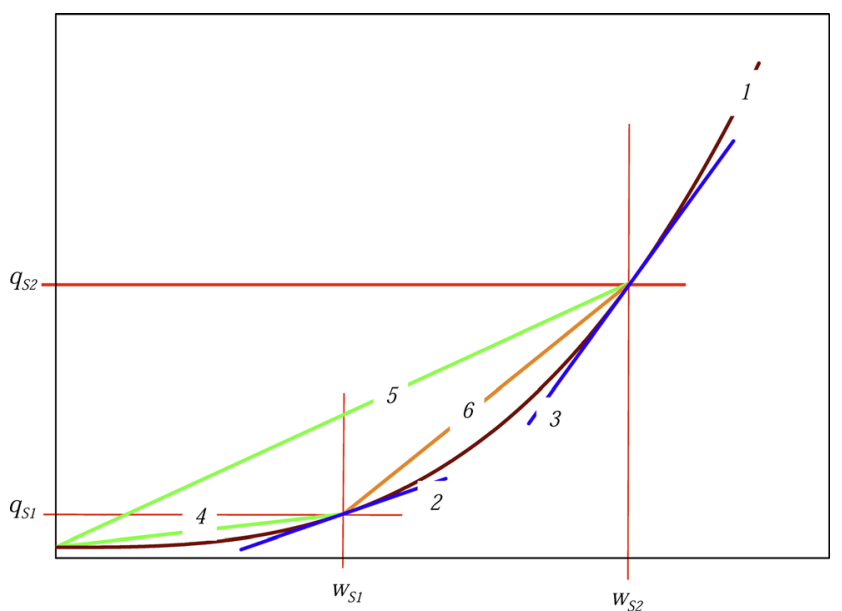

FIGURE 5. Kinematic flow relationships. Volume flux densities $q_{\mathrm{s} 1}$ $\left(w_{S_{1}}\right)$ and $q_{\mathrm{S}_{2}}\left(w_{\mathrm{S}_{2}}\right)$ of $w_{\mathrm{S} 1}<w_{\mathrm{S} 2}$ under the macropore flow restriction. Line (1): volume flux density versus mobile water content $q(w)$; lines (2) and (3): the slopes of the tangents represent the draining front celerities $C_{\mathrm{D} 1}$ and $C_{\mathrm{D} 2}$; lines (4) and (5): the slopes of the chords $q / w$ represent the wetting shock front velocities $v_{1}$ and $v_{2}$; line (6): the slope of the chord represents the celerity $c_{J 12}$ of the jump from $w_{S 2}$ to $w_{S 1}$. Adapted with permission from Germann (2014). A color version of this figure is available in the online version of this article. futcolore

\section{Propagation of a Single Pulse}

Let's consider one pulse $P\left(q_{S}, T_{B}, T_{E}\right)$, hence, with $w_{S}$ according to Eq. [34]. The interception time $T_{\mathrm{I}}$ separates pulse routing in an early interval, $T_{B} \leq \tau_{1} \leq T_{\mathrm{I}}$, and in late interval, $\tau_{2} \geq T_{\mathrm{I}}$, whereas $q(z, t)$ or $w(z, t)$ are the WCW properties to be routed in the two intervals.

\section{(i) Interval $T_{B} \leq \tau_{1} \leq T_{\mathrm{I}}$}

Interception depth and time are the result from the same procedure that led to Eqs. [11] and [12] as Fig. 6 illustrates: line (1) represents the position of the wetting shock front, and line (3) the characteristic (i.e., trajectory) of the draining front (for clarity, Fig. 6 omits labeling $\mathrm{Z}_{\mathrm{I}}$ ). The figure also compares the wetting shock front depth of a pulse produced by a smaller mobile water content $w_{\mathrm{S} 1 / 2}$ (thin lines) with the wetting shock front depth of a pulse produced by a greater mobile water content $w_{S}$ (heavy lines), thus demonstrating the exclusive dependence of $T_{\mathrm{I}}$ on $T_{B}$ and $T_{E}$ and independent from $w_{S}$ and $q_{S}$.

During $\tau_{1}$, all the laminae of $P$ move along their characteristics that are intercepted by the slower-moving wetting shock front. The slopes of the characteristics express the laminae's celerities as lines (2) and (3) in Fig. 6 as well as lines (2) and (3) in Fig. 5 illustrate. The mobile water content along the wetting shock front is constant at $w_{S}$.

\section{(ii) Interval $\tau_{2} \geq T_{1}$}

The cessation of input at $T_{E}$ releases at once all the rear ends of the laminae, whereas the lamina at $F$, Figs. 1 and 2, moves the fastest with $c_{\mathrm{D}}=c(f)$, Eq. [2], along the characteristic of line (3) in Fig. 6. Each rear end moves with the celerity $c(f)$ that decreases with decreasing distance $f$ from the SWI (Fig. 1). Thus, the film starts to collapse, the WCW flattens, and the characteristics spread as, for instance, line (4) in Fig. 6 indicates. Spreading of the characteristics implies deceleration of the wetting shock front whose position $z_{W}\left(\tau_{2}\right)$ is, in analogy with Eq. [21],

$$
z_{W}\left(\tau_{2}\right)=c_{D} \cdot\left(\frac{T_{E}-T_{B}}{2}\right)^{2 / 3} \cdot\left(\tau_{2}-T_{E}\right)^{1 / 3}
$$

The mobile water content along the wetting shock front is similar to Eq. [29], while $w(z, t)$ in the area confined by the characteristic of the draining front, line (3) in Fig. 6 and Eq. [18], and the wetting shock front, Eq. [39], are according to Eq. [27].

Germann (2014) demonstrated that the straight-lined position of the wetting shock front, $z_{W}\left(\tau_{1}\right)$, prior to $T_{\mathrm{I}}$, line (1) in Fig. 6, generally touches tangentially at the interception point $\left(Z_{\mathrm{I}} / T_{\mathrm{I}}\right)$ the curvedlined position $z_{W}\left(\tau_{2}\right)$ of the wetting shock front after $T_{\mathrm{I}}$ line (5) in Fig. 6. Thus Eqs. [21] and [39] express the pulse's interception function that is completely determined by $c_{\mathrm{D}}$, Eq. [10], and the pulse duration $\left[T_{E}-T_{B},\right]$. Accordingly, line (5) in Fig. 6 represents the potential position of interception $\left(Z_{\mathrm{I}} / T_{\mathrm{I}}\right)$ prior to $T_{\mathrm{I}}$ that turns into the effective wetting shock front position $z_{W}\left(\tau_{2}\right)$ after $T_{\mathrm{I}}$. The interception function facilitates modeling.

\section{Propagation of a Faster Pulse With Higher-Volume Flux Density Trailing a Slower Pulse With Lower-Volume Flux Density}

Let's now consider two pulses $P_{1}\left(q_{\mathrm{S} 1}, T_{\mathrm{B} 1}, T_{\mathrm{E} 1}\right)$ and $P_{2}\left(q_{\mathrm{S} 2}, T_{\mathrm{B} 2}\right.$, $\left.T_{\mathrm{E} 2}\right)$, where $q_{\mathrm{S} 2}<q_{\mathrm{S} 1}, w_{\mathrm{S} 2}<w_{\mathrm{S} 1}$, and $T_{\mathrm{E} 1}=T_{\mathrm{B} 2}$; thus, $P_{1}$ trails $P_{2}$. As shown in Fig. 7, the arrangement requires three interception depths and times that are

$T_{\mathrm{I} 1}$, when the wetting shock front of $P_{1}$ intercepts its draining front, lines (1) and (2) in Fig. 7;

$T_{\mathrm{I} 12}$, when the wetting shock front of $P_{1}$ intercepts the draining front of the first lamina of $P_{2}$, as expressed by its characteristic, line (3) in Fig. 7; and

$T_{\text {I2 }}$, when the straight wetting shock front of $P_{2}$ intercepts the draining from the last lamina of $P_{2}$ as shown with its characteristic, line (4) in Fig. 7. Lines (3) and (4) are parallels.

The following presents the procedures of determining the three interception times and depths, the wetting shock front depths during the four intervals of(i) $\left[T_{B} \leq \tau_{1} \leq T_{\mathrm{I} 1}\right]$, (ii) $\left[T_{\mathrm{I} 1} \leq \tau_{2} \leq T_{\mathrm{I} 12}\right]$, (iii) $\left[T_{\mathrm{I} 12} \leq \tau_{3} \leq T_{\mathrm{I} 2}\right]$, (iv) $\left[\tau_{4} \geq T_{\mathrm{I} 2}\right]$, and the associated mobile water contents.

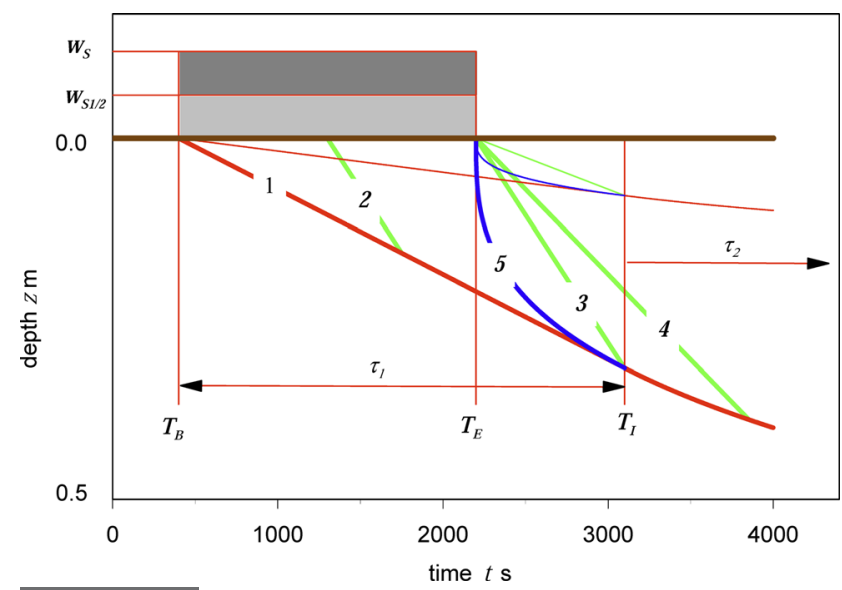

FIGURE 6. Wetting front trajectory, characteristics, and interception function of two single pulses. $w_{S}$ and $w_{S 1 / 2}$ are the mobile water contents resulting from two pulses with the same beginning at $T_{\mathrm{B}}$ and ending at $T_{\mathrm{E}}$. The heavy lines are related to $w_{\mathrm{S}}$, whereas the thin lines represent $w_{S 1 / 2}$. The wetting front, line (1), cuts the characteristic of the draining front, line (3) at the time of interception, $T_{1}$; line (2) is the characteristic of a arbitrary lamina released at time $t<T_{\mathrm{E}}$ that is parallel to line (3); line (4) is the characteristic of an arbitrary lamina that was released at $f<F$ at $t=T_{\mathrm{E}}$; line (5) is the interception function (that turns into the curved temporal position of the wetting shock front during $\tau_{2}$ ); the interception time $T_{1}$ separates the periods $\tau_{1}$ and $\tau_{2}$, whereas it is independent from the water contents of the pulses. (The depth $Z_{1}$ corresponding to $T_{1}$ is not marked for clarity reasons.) Adapted with permission from Germann (2014). See also Fig. 5 for the KW expressions. A color version of this figure is available in the online version of this article. foun color 


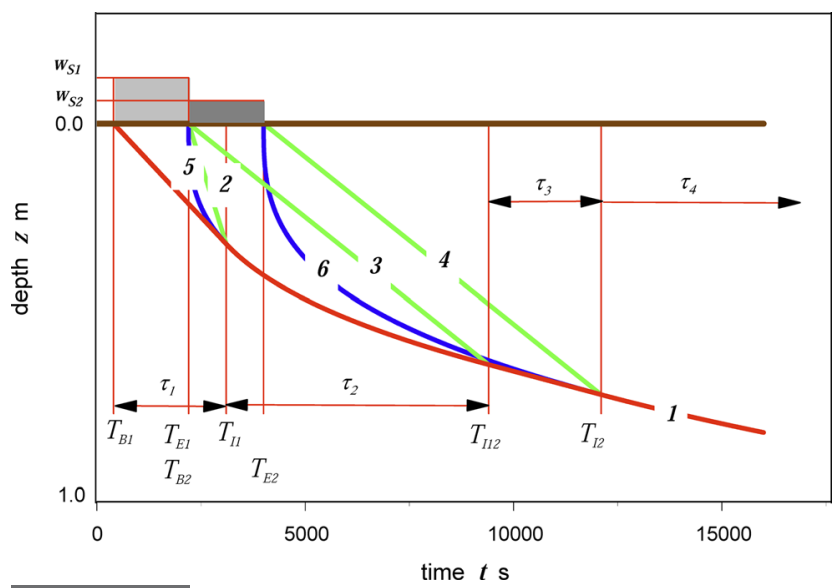

FIGURE 7. Wetting front trajectory, characteristics, and interception function of the faster pulse $P_{1}$ trailing the slower pulse $P_{2}$. Line (1) gives the temporal position of the wetting front during the four periods $\tau_{1}$ to $\tau_{4}$ between the beginning of $P_{1}$ at $T_{\mathrm{B} 1}$, the interception times $T_{11}, T_{112}, T_{12}$, and after $T_{12}$; line (2) is the characteristic of the draining front from $P_{1}$; lines (3) and (4) are the parallel characteristics of $P_{2}$; lines (5) and (6) are the interception functions (that turn into the curved sections of the wetting shock fronts during the respective periods $\tau_{2}$ and $\tau_{4}$.) Adapted with permission from Germann (2014). See also Fig. 5 for the KW expressions. A color version of this figure is available in the online version of this article. founcolore

\section{(i) Interval $T_{B} \leq \tau_{1} \leq T_{11}$}

The wetting shock front of $P_{1}$, line (1) in Fig. 7, intercepts $P_{1}$ 's draining front, line (2) in Fig. 7, at $T_{\mathrm{I} 1}$ and $Z_{\mathrm{I} 1}$ that are, in accord with Eqs. [11] and [12],

$$
\begin{aligned}
& T_{I 1}=\frac{1}{2} \cdot\left(3 \cdot T_{E 1}-T_{B 1}\right) \\
& Z_{I 1}=\frac{c_{D 1}}{2} \cdot\left(T_{E 1}-T_{B 1}\right)
\end{aligned}
$$

where $c_{\mathrm{D} 1}$ expresses the celerity of $P_{1}$ 's draining front that started moving at $T_{\mathrm{E} 1}$. During $\tau_{1}$, the mobile water content at the wetting front amounts to $w_{\mathrm{S} 1}$ that is maintained between the wetting and draining fronts, lines (1) and (2) in Fig. 7. After passing of the draining front, the mobile water content reduces in analogy to Eq. [27].

\section{(ii) Interval $T_{\mathrm{I} 1} \leq \tau_{2} \leq T_{\mathrm{I} 12}$}

Line (5) in Fig. 7 shows the interception function of $P_{1}$ that continues, in analogy with Eq. [21], as curved wetting shock front during $\tau_{2}$ as

$$
z_{W}\left(\tau_{2}\right)=c_{D 1} \cdot\left(\frac{T_{E 1}-T_{B 1}}{2}\right)^{2 / 3} \cdot\left(\tau_{2}-T_{E 1}\right)^{1 / 3}
$$

that is, line (1) in Fig. 7. However, only the rear ends of $P_{1}$ 's laminae in the mobile water content range of $w_{\mathrm{S} 1}>w>w_{\mathrm{S} 2}$ arrive at the wetting front, whereas those of $w<w_{\mathrm{S} 2}$ do not show because $P_{2}$ sustains $w_{\mathrm{S} 2}$. While $P_{1}$ trails $P_{2}$, the first lamina of $P_{2}$ moves with the celerity $c_{\mathrm{D} 2}$ (i.e., $P_{1}$ lubricates $\left.P_{2}\right)$. Thus, the wetting shock front $z_{W}\left(\tau_{2}\right)$, Eq. [42], intercepts at $\left(Z_{\mathrm{I} 12} / T_{\mathrm{I} 12}\right)$, the first lamina of $P_{2}$ whose characteristic is line (3) in Fig. 7. Equating the temporal position of
$c_{\mathrm{D} 2} \times\left(\tau_{2}-T_{\mathrm{B} 2}\right)$ of $P_{2}$ 's first lamina with Eq. [42] and solving for time lead to the interception time and depth of

$$
\begin{gathered}
T_{I 12}=T_{B 2}+\left(\frac{c_{D 1}}{c_{D 2}}\right)^{3 / 2} \cdot \frac{\left(T_{B 2}-T_{B 1}\right)}{2} \\
Z_{I 12}=c_{D 2} \cdot\left(\frac{c_{D 1}}{c_{D 2}}\right)^{3 / 2} \cdot \frac{\left(T_{B 2}-T_{B 1}\right)}{2}
\end{gathered}
$$

During $\tau_{2}$, the mobile water content at the wetting shock front gradually reduces from $w_{\mathrm{S} 1}$ to $w_{\mathrm{S} 2}$ in accord with Eq. [29]

$$
w\left(\tau_{2}\right)=w_{S 1} \cdot\left(\frac{T_{E 1}-T_{B 1}}{2 \cdot\left(\tau_{2}-T_{E 1}\right)}\right)^{1 / 3}
$$

From the surface to the wetting front, the mobile water content reduces from $w_{\mathrm{S} 1}$ to $w_{\mathrm{S} 2}$ between the characteristic of $P_{1}$ 's draining front, line (2), and $P_{2}$ 's first lamina, line (3) in Fig. 7, where $w\left(\tau_{2}\right)$ from Eq. [45] replaces $w_{\mathrm{CR}}$ in Eq. [29].

\section{(iii) Interval $T_{\mathrm{I} 12} \leq \tau_{3} \leq T_{\mathrm{I} 2}$}

The laminae of $P_{2}$ glide one over the other with constant celerity that results in the constant wetting shock front velocity of $v_{\mathrm{W} 2}=c_{\mathrm{D} 2} / 3$. Thus, the temporal wetting shock front depth during $\tau_{3}$ becomes

$$
z_{W}\left(\tau_{3}\right)=v_{W 2} \cdot\left(\tau_{3}-T_{B 2 a}\right)
$$

where the time offset $T_{\mathrm{B} 2 \mathrm{a}} \mathrm{s}$ indicates the apparent earlier beginning of $P_{2}$ that accounts for $P_{1}$ trailing $P_{2}$ (i.e., $P_{2}$ pick-a-packing on $P_{1}$ ). Thus, the wetting front of $P_{2}$ appears earlier at $Z_{\mathrm{I} 12}$ than if it were released from and moving as a single pulse. Thus, $T_{\mathrm{B} 2 \mathrm{a}}<T_{\mathrm{B} 2}$, and

$$
T_{B 2 a}=T_{I 12}-\frac{Z_{I 12}}{v_{W 2}}
$$

The time offset $T_{\mathrm{B} 2 a}$ affects only the temporal position of the wetting front, but by no means does it affect the WCWs' volume balances. Also, the characteristic of $P_{1}$ 's draining front, line (2) in Fig. 7, is steeper than the characteristics of $P_{2}$ 's laminae, lines $(3,4)$ in Fig. 7. This again is due to $P_{1}$ 's trailing of $P_{2}$. (See also the corresponding slopes of lines (5) and (4) on $q(w)$ in Fig. 5 that indicate the slowdown of the fronts when switching from $w_{\mathrm{S} 2}$ to $w_{\mathrm{S} 1}$.) During $\tau_{3}$, the draining front of $P_{2}$ moves along the characteristic $z_{\mathrm{D} 2}\left(\tau_{3}\right)$, line (4) in Fig. 7, that is

$$
z_{D 2}\left(\tau_{3}\right)=c_{D 2} \cdot\left(\tau_{3}-T_{E 2}\right)
$$

Equating Eq. [46] with Eq. [48] and solving for $\tau_{3}$ lead to the interception time and depth of

$$
\begin{aligned}
& T_{I 2}=\frac{1}{2} \cdot\left(\frac{Z_{I 12}}{v_{W 2}}+3 \cdot T_{\mathrm{E} 2}-T_{I 12}\right) \\
& Z_{I 2}=\frac{3}{2} \cdot\left[Z_{I 12}+v_{W 2} \cdot\left(T_{E 2}-T_{I 12}\right)\right]
\end{aligned}
$$

During $\tau_{3}$, the mobile water content at the wetting front amounts to $w_{\mathrm{S} 2}$. It remains at $w_{\mathrm{S} 2}$ between the characteristics of $P_{2}$ 's first and last laminae, lines (3) and (4) in Fig. 7.

\section{(iv) Interval $\tau_{4} \geq T_{12}$}

In analogy with Eqs. [21] and [42], during $\tau_{4}$ and below $Z_{\mathrm{I} 2}$, the wetting front $z_{W}\left(\tau_{4}\right)$ moves along line (1) in Fig. 7, as 


$$
z_{W}\left(\tau_{4}\right)=c_{D 2} \cdot\left(\frac{T_{E 2}-T_{B 2 a}}{2}\right)^{2 / 3} \cdot\left(\tau_{4}-T_{E 2}\right)^{1 / 3}
$$

The mobile water content at the wetting front during $\tau_{4}$ amounts to

$$
w\left(\tau_{4}\right)=w_{S 2} \cdot\left(\frac{T_{E 2}-T_{B 2}}{2 \cdot\left(\tau_{4}-T_{E 2}\right)}\right)^{1 / 3}
$$

where $w\left(\tau_{4}\right)$ from Eq. [52] replaces $w_{\mathrm{CR}}$ in Eq. [29]. The mobile water content in the spatiotemporal range beyond the characteristic of the last lamina of $P_{2}$ (i.e., to the right of line (4) in Fig. 7) follows from Eq. [27], where $w_{\mathrm{S} 2}$ replaces $w_{S}$.

All volume flux densities are functions of the mobile water contents according to Eq. [32]. The water balance of the two pulses $P_{1}$ and $P_{2}$ at any given time $t>T_{\mathrm{B} 1}$ equates the total volume of infiltrated water with the total increase of the mobile water content from the soil surface to the wetting shock front depth at $z_{W}(t)$. For example, the total volume of the infiltrated water must be equal to the total increase of the mobile water content from the surface to the wetting shock front depth after its interception of the second draining front at depths $z_{W}\left(\tau_{4}\right)$, thus

$$
q_{S 1} \cdot\left(T_{E 1}-T_{B 1}\right)+q_{\mathrm{S} 2} \cdot\left(T_{E 2}-T_{B 2}\right)=\int_{0}^{z_{W}(\tau 4)} w(z, t) \mathrm{d} z=\frac{2}{3} \cdot L \cdot\left(\frac{\eta}{g}\right)^{\frac{1}{2}} \cdot\left(\tau_{4}-T_{E 2}\right) \cdot z_{W}\left(\tau_{4}\right)^{\frac{3}{2}}[53]
$$

The right-hand side of Eq. [53] results from integrating the water content profile, Eq. [22], where the depth $z_{W}\left(\tau_{4}\right)$ follows from Eq. [51].

\section{Propagation of a Faster Pulse With Higher-Volume Flux Density That Superimposes (Overtakes) a Slower Pulse With Lower-Volume Flux Density}

Let's consider two pulses $P_{1}\left(q_{1}, T_{\mathrm{B} 1}, T_{\mathrm{E} 1}\right)$ and $P_{2}\left(q_{2}, T_{\mathrm{B} 2}, T_{\mathrm{E} 2}\right)$, such that $q_{\mathrm{S} 2}>q_{\mathrm{S} 1}, w_{\mathrm{S} 2}>w_{\mathrm{S} 1}$, and $T_{\mathrm{E} 1}=T_{\mathrm{B} 2}$. Mass balance requires the discontinuity of the jump from $P_{1}$ to $P_{2}$ to move with the celerity of

$$
c_{J 12}=\frac{q_{S 2}-q_{S 1}}{w_{2}-w_{1}}
$$

$\mathrm{m} \cdot \mathrm{s}^{-1}$ (Lighthill and Witham, 1955). Line (2) in Fig. 8 shows the characteristic of the jump. (The slope of line (6) in Fig. 5 represents $c_{J 12}$.) Figure 8 suggests two interception times:

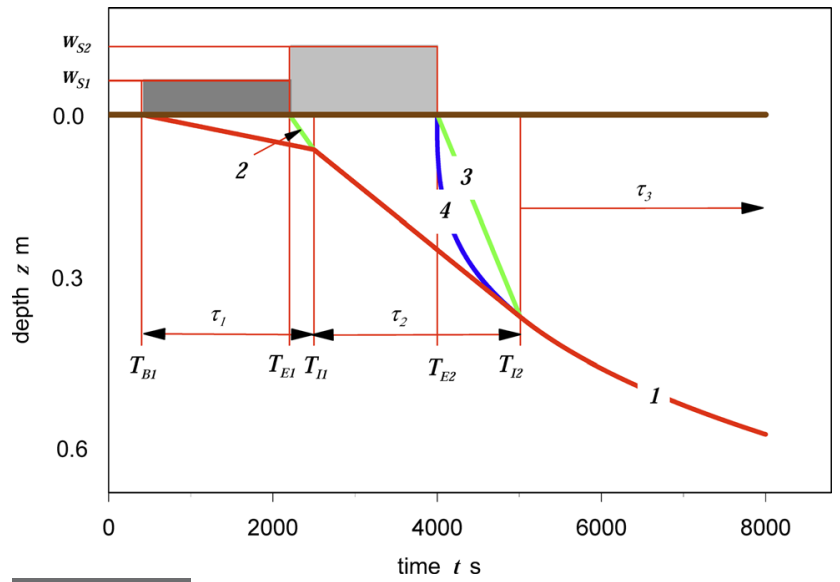

FIGURE 8. Wetting front trajectory, characteristics, and interception function of the faster pulse $P_{2}$ superimposing (overtaking) the slower pulse $P_{1}$. Line (1) gives the temporal position of the wetting shock front during the three periods $\tau_{1}$ to $\tau_{3}$ between the beginning of $P_{1}$ at $T_{\mathrm{B} 1}$, the interception times $T_{11}, T_{12}$, and after $T_{12}$; lines (2) and (3) are the characteristics of the jump from $P_{1}$ to $P_{2}$ and the draining front of $P_{2}$ respectively; line (4) is the interception function (that turns into the curved section of the wetting shock front after $T_{12}$ ). Adapted with permission from Germann (2014). See also Fig. 5 for the KW expressions. A color version of this figure is available in the online version of this article.
$T_{\text {I1 }}$, when the wetting shock front of $P_{1}$, line (1) in Fig. 8, intercepts the jump characteristic, line (2) in Fig. 8, and $T_{\mathrm{I} 2}$, when the wetting shock front from the combined $P_{1}$ and $P_{2}$ intercepts the draining from the last lamina of $P_{2}$, characteristic line (3) in Fig. 8.

The following presents the procedures of determining the two interception depths and times, the spatiotemporal position of the wetting shock front in the three intervals of(i) $\left[T_{B} \leq \tau_{1} \leq T_{\mathrm{I} 1}\right]$, (ii) $\left[T_{\mathrm{I} 1} \leq \tau_{2} \leq T_{\mathrm{I} 2}\right]$, (iii) $\left[\tau_{3} \geq T_{\mathrm{I} 2}\right]$, and the associated mobile water contents.

\section{(i) Interval $T_{\mathrm{B} 1} \leq \tau_{1} \leq T_{\mathrm{I} 1}$}

The wetting shock front of $P_{1}$ moves with $v_{\mathrm{W} 1}$, Eq. [7] and line (1) in Fig. 8. It intercepts the characteristic of the jump, Eq. [54] and line (2) in Fig. 8, at time and depth of $T_{\mathrm{I} 1}$ and $Z_{\mathrm{I} 1}$. Solving the pair of linear equations of $Z_{\mathrm{I} 1}=\left(T_{\mathrm{I} 1}-T_{\mathrm{B} 1}\right) \cdot v_{\mathrm{W} 1}$ and $Z_{I 1}=\left(T_{I 1}-T_{E 1}\right) \cdot c_{J 12}$ yields

$$
\begin{aligned}
T_{I 1} & =\frac{T_{E 1} \cdot c_{J 12}-T_{B 1} \cdot v_{W 1}}{c_{J 12}-v_{W 1}} \\
Z_{I 1} & =c_{J 12} \cdot v_{W 1} \cdot \frac{T_{E 1}-T_{B 1}}{c_{J 12}-v_{W 1}}
\end{aligned}
$$

The mobile water content at the wetting front during $\tau_{1}$ is $w_{\mathrm{S} 1}$ that also applies to the triangle between the surface, the wetting front, and the characteristic of the jump.

\section{(ii) Interval $T_{11} \leq \tau_{2} \leq T_{12}$}

Beyond $Z_{\mathrm{I} 1}$ and after $T_{\mathrm{I} 1}$, the wetting front moves with the velocity $v_{\mathrm{W} 2}$ according to $P_{2}$, slope of line (1) in Fig. 8 , with the characteristic of

$$
z_{W}\left(\tau_{2}\right)=\left(\tau_{2}-T_{B 2 a}\right) \cdot v_{W 2}
$$

Again, the offset of $T_{\mathrm{B} 2 \mathrm{a}}$, Eq. [47], indicates the apparent earlier release of $P_{2}$ because of its gliding on $P_{1}$. The draining front of $P_{2}$ is released at $T_{\mathrm{E} 2}$ with the characteristic of line (3) in Fig. 8. Line (3) is steeper than line (2), indicating that $P_{2}$ superimposes $P_{1}$. The wetting front of $P_{2}$, line (1) in Fig. 8, intercepts $P_{2}$ 's draining front at $T_{\mathrm{I} 2}$ and $Z_{\mathrm{I} 2}$. Solving the pair of linear equations of $Z_{I 2}=Z_{I 1}+\left(T_{I 2}-T_{I 1}\right) \cdot v_{W 2}$ and $Z_{\mathrm{I} 2}=\left(T_{\mathrm{I} 2}-T_{\mathrm{E} 2}\right) \cdot c_{\mathrm{D} 2}$, while recognizing that $c_{\mathrm{D} 2}=3 \times v_{\mathrm{W} 2}$, yields

$$
\begin{aligned}
& T_{I 2}=\frac{1}{2} \cdot\left(3 \cdot T_{E 2}-T_{B 2 a}\right) \\
& Z_{I 2}=\frac{c_{D 2}}{2} \cdot\left(T_{E 2}-T_{B 2 a}\right)
\end{aligned}
$$

The mobile water content at the wetting front is $w_{\mathrm{S} 2}$ as well as in the spatiotemporal quadrangle between the surface, the characteristic of the jump, line (2); the wetting front, line (1); and the characteristic of the draining front, line (3).

\section{(iii) Interval $\tau_{3} \geq T_{12}$}

The interception function is released at $T_{\mathrm{E} 2}$, which appears as curved wetting front depth during $\tau_{3}$, line (1) in Fig. 8. It progresses according to Eq. [21] as

$$
z_{W}\left(\tau_{3}\right)=c_{D 2} \cdot\left(\frac{T_{E 2}-T_{B 2 a}}{2}\right)^{2 / 3} \cdot\left(\tau_{3}-T_{E 2}\right)^{1 / 3}
$$

After passing of the draining front, the mobile water content decreases according to Eq. [27]. Alluding to Eq. [29], the mobile water content along the wetting front during $\tau_{3}$, line (1) in Fig. 8, amounts to

$$
w\left(\tau_{3}\right)=w_{S 2} \cdot\left(\frac{T_{E 2}-T_{B 2}}{2 \cdot\left(\tau_{3}-T_{E 2}\right)}\right)^{1 / 3}
$$




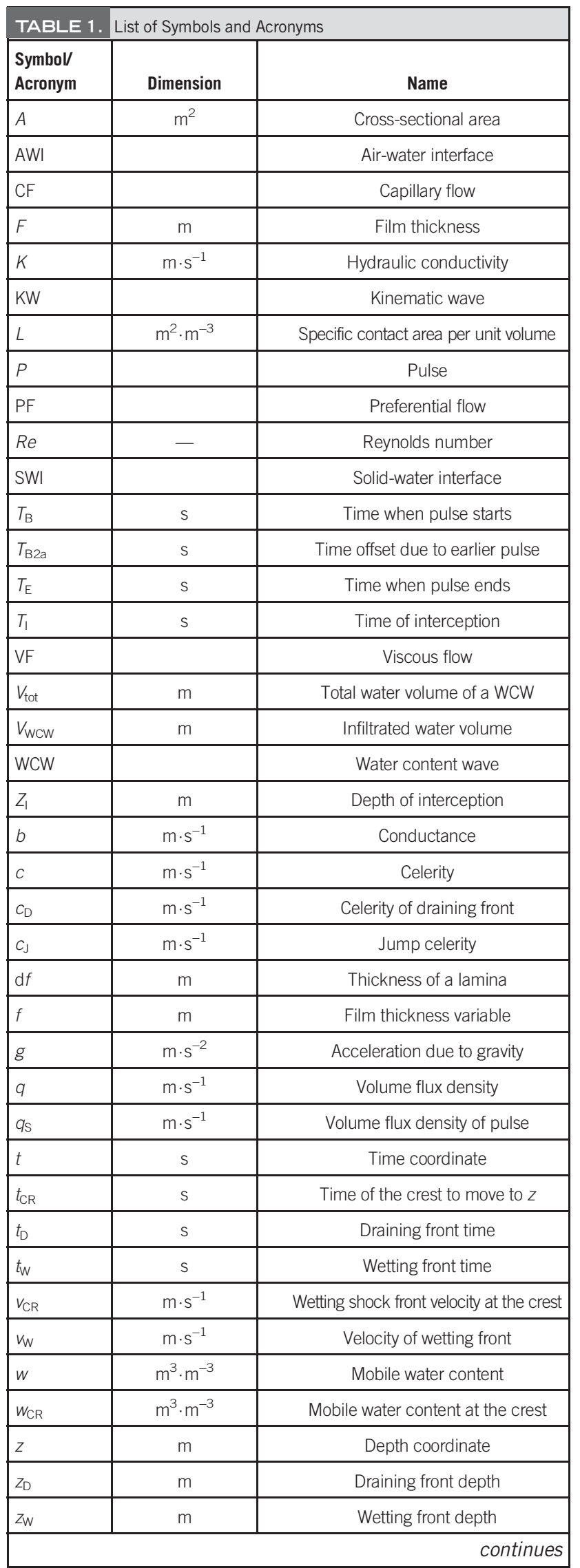

\begin{tabular}{|c|c|c|}
\hline $\begin{array}{l}\text { Symbol/ } \\
\text { Acronym }\end{array}$ & Dimension & Name \\
\hline$\zeta$ & $\mathrm{m}$ & Local depth variable \\
\hline$\eta$ & $\mathrm{m}^{2} \cdot \mathrm{s}^{-1}$ & Kinematic viscosity \\
\hline$\theta$ & $m^{3} \cdot m^{-3}$ & Volumetric water content \\
\hline$\rho$ & $\mathrm{kg} \cdot \mathrm{m}^{-3}$ & Density \\
\hline$\tau$ & s & Local time variable \\
\hline$\varphi$ & $\mathrm{Pa}$ & Shear force \\
\hline$\psi$ & $\mathrm{Pa}$ & Capillary potential \\
\hline
\end{tabular}

During $\tau_{3}$, the mobile water content behind the wetting front evolves according to Eq. [31], where $w\left(\tau_{3}\right)$ from Eq. [61] replaces $w_{\mathrm{CR}}\left(\zeta_{2}, t\right)$.

All volume flux densities follow from the mobile water contents according to Eq. [32]. The water balance calculations follow from Eq. [53].

\section{Discussion of Characteristics (i.e., Trajectories) and Kinematic Waves}

Kinematic wave theory provides a robust mathematical tool for routing wetting shock fronts originating from individual pulses that belong to extended time series. So far, the approach is limited to the macropore flow restriction, Eq. [35]; however, there is confidence in the development of experimental relationships of $L\left(q_{S}\right)$. The $w(z, t)$ and $q(z, t)$ values can be grafted on the temporal positions of the wetting shock fronts and draining fronts once the interception depths and times have been determined. Pulse routing remains robust also in the cases of either $w_{1}=w_{2}$ or $w_{2}=0$, thus rendering even more flexibility to the modeling of PF (Table 1).

\section{SUMMARY, DISCUSSION, AND CONCLUSIONS}

Newton's (1729) law of shear is the base for momentum dissipation during laminar flow that evolves here as VF to the approaching of $\mathrm{PF}$ in permeable media. The concept exclusively uses analytical expressions that result in a set of stringent theoretical relationships completely expressing PF. Superfluous numerical procedures neither obscure the VF approach, nor do they introduce ambiguity. Thus, the spatiotemporal propagation of an input pulse depends only on the two parameters, film thickness $F$ and specific contact area $L$, greatly facilitating calibration and application. Because viscosity continuously balances gravity, the concept of representative elementary volume, REV, is redundant. Therefore, scale restrictions of the spatiotemporal approach's applicability vanish. The WCW emerges as the basic unit of the spatiotemporal distribution of mobile water content that is due to the infiltration of a rectangular pulse. Analytical expressions define adequately the wave's projections onto the two planes of mobile water content versus time and depth, respectively. Further, the wave's projection onto the depth-time plane links the approach with the theory of KW according to Lighthill and Witham (1955). The link thus leads to the routing of input pulse series, each pulse with individual duration and intensity. The twopulse examples provided in "Propagation of a Faster Pulse With Higher-Volume Flux Density That Superimposes (Overtakes) a Slower Pulse With Lower-Volume Flux Density" and "Discussion of Characteristics (i.e., Trajectories) and Kinematic Waves" extend to their respective limits of $q_{\mathrm{S} 1}=q_{\mathrm{S} 2}$, which greatly facilitates modeling of pulse series.

Already the assessment of the pulses' penetration depths indicates a wide spectrum of VF applications. For instance, shallow penetrations of short pulses are important in the optimization of irrigation schemes, whereas longer lasting pulses reaching greater depths are 
important in groundwater recharge considerations. Thus, adaption to specific problems is simple because the approach applies to both situations without scale jump. From the approach's stringency follows that any deviations from water balance calculations, Eq. [53], are exclusively due to either methodological uncertainties or to deviations from VF and PF in view of this contribution. Methodological uncertainties set aside, too low a depth integral of mobile water content in comparison with the volume of infiltration indicates on the one hand flow divergence within a volume of the permeable medium that is considerably larger than the control volume occupied by the instrumentation. On the other hand, it may indicate water abstraction from the WCW due to capillarity. Observed deviations thus provide for a starting point for investigating interactions between the $F$ und $L$ parameters on one side and the spatiotemporal action of capillarity on the other side. Conversely, too high a depth integral of mobile water in the balance is indicative of flow converging from a volume of the permeable medium larger than the control volume of instrumentation. Investigating balance deviations due to flow divergence or convergence lead to spatial variability considerations of PF. Also, volume balance considerations in view of VF may help understanding nonequilibrium flow in Richards' (1931) CF (Germann, 2018a).

However, the VF approach to PF is limited to laminar flow as expressed with the Reynolds number, Eq. [8]. It is further restricted to gravity-driven flow, that is, close to the vertical-down direction. Thus, VF excludes a priori any capillary rise. For practical reasons, the macropore flow restriction, Eq. [35], has to be assumed as long as no relationships between volume flux density of input and $L$ have been explored in more details.

Scientifically accepted theory and experimental procedures are available for the in situ determination of the VF parameters, film thickness $F$ and specific contact area $L$ (Germann, 2018b). Thus, the VF package just awaits to be used for addressing various PF problems. For example, the specific contact area $L$ is considered the locus of momentum dissipation, exchange of heat, solutes, and particles between PF and the resting parts of the permeable medium. In particular, $L$ seems the predestined parameter of abstraction from mobile water due to capillarity.

\section{ACKNOWLEDGMENT}

The editor-in-chief of Soil Science, Daniel Gimenez, invited the manuscript on hydromechanics and kinematics in PF. His and Tammo Steenhuis' comments greatly improved the revision.

\section{REFERENCES}

Beven K., and P. Germann. 1981. Water flow in soil macropores II. A combined flow model. J. Soil Sci. 32(1):15-29.

Darcy H. 1856. Les fontaines publiques de la ville de Dijon. Dalmont, Paris.
Dupuit J. 1863. Etudes théoriques et pratiques sur le mouvement des eaux dans les canaux découverts et à travers les terrains perméables. ((deuxième édition) ed.) Dunod, Paris.

Germann P. F. 1985. Kinematic wave approach to infiltration and drainage into and from soil macropores. Trans. ASAE. 28(3):745-749.

Germann P. F. 1986. Rapid drainage response to precipitation. Hydrol. Process. 1:2-13.

Germann P. F. 2014. Preferential Flow-Stokes Approach to Infiltration and Drainage. Geographica Bernensia, University of Bern, Bern, Switzerland.

Germann P. F. 2018a. Viscosity - the weak link between Darcy's law and Richards' (1931) capillary flow. Hydrol. Process. 32:1166-1172.

Germann P. F. 2018b. Preferential flow at the Darcy-scale: Parameters from water content time series. Methods Soil Anal. 3:160121.

Germann P. F., and S. A. al-Hagrey. 2008. Gravity-driven and viscositydominated infiltration into a full-scale sand model. Vadose Zone J. 7:1160-1169

Germann P. F., and M. Karlen. 2016. Viscous-flow approach to in situ infiltration and in vitro hydraulic conductivity determination. Vadose Zone J. 15(12).

Germann P. F., and V. Prasuhn. 2017. Viscous flow approach to rapid infiltration and drainage in a weighing lysimeter. Vadose Zone J. 17:170020.

Germann P. F., W. M. Edwards, and L. B. Owens. 1984. Profiles of bromide and increased soil moisture after infiltration into soils with macropores. Soil Sci. Soc. Am. J. 48(2):237-244.

Harrold L. L., and F. R. Dreibelbiss. 1958. Evaluation of agricultural hydrology by monolith lysimeters. In: USDA Tech. Bull. No. 1179, pp. 166.

Hincapié I. A., and P. Germann. 2009a. Abstraction from infiltrating water content waves during weak viscous flows. Vadose Zone J. 8:996-1003.

Hincapié I., and P. F. Germann. 2009b. Impact of initial and boundary conditions on preferential flow. J. Contam. Hydrol. 104:67-73.

Hincapié I. A., and P. Germann. 2010. Water content wave approach applied to neutron radiographs of finger flow. Vadose Zone J. 9:278-284.

Jarvis N., J. Koestel, and M. Larsbo. 2016. Understanding preferential flow in the vadose zone: Recent advances and future prospects. Vadose Zone J. 15(12).

Kelley G. P., W. M. Edwards, L. L. Harrold, and J. L. McGuiness. 1975. Soils of the North Appalachian Watershed. USDA Misc. Publ. No. 1296. U.S. Government Priniting Office, Washington, DC.

Lawes J. B., J. H. Gilbert, and R. Warington. 1882. On the amount and composition of the rain and drainage waters collected at Rothamsted. W. Clowes and Sons, Ltd., London.

Lighthill M. J., and G. B. Witham. 1955. On kinematic waves-I. Flood movement in long rivers. Proc. R. Soc. London Ser. A. 229:281-316.

Newton I. 1729. The Mathematical Principles of Natural PhilosophyTranslation Into English. Vol. II. Benjamin Motte, London, UK, pp. 184.

Nimmo J. R. 2010. Theory of source-responsive and free-surface film modeling of unsaturated flow. Vadose Zone J. 9:295-306.

Nimmo J. R. 2012. Preferential flow occurs in unsaturated conditions. Hydrol. Process. 26:786-789.

Poiseuille J. L. M. 1846. Recherches expérimentales sur le mouvement des liquides dans les tubes de très petits diamètres. Comptes Rendus, xi-xii. Mém. des Sav. Etrangers, ix.

Richards L. A. 1931. Capillary conduction of liquids through porous mediums. Phys. Chem. Chem. Phys. 1:318-333. 